\title{
Las preferencias participativas del profesorado de educación física en su actividad profesional
}

\author{
Maria I. Barriopedro*, Luis M. Ruiz-Pérez, Miguel A. Gómez-Ruano e Isabel Rico
}

Universidad Politécnica de Madrid (España).

\begin{abstract}
Resumen: Los entornos colaborativos han sido promocionados como la mejor solución a los problemas que la actividad docente puede presentar independientemente de las preferencias personales de los profesores. El objetivo del estudio es desarrollar una escala de preferencias de interacción social en el entorno laboral para los profesores de educación física, partiendo de la Escala GR de Ruiz et al. (2010), así como evaluar su equivalencia de medida en profesores con diferentes años de experiencia docente y entre hombres y mujeres, utilizando el análisis factorial confirmatorio multigrupo. En este estudio participaron 1413 profesores españoles. La escala final quedó constituida por las 4 cuatro dimensiones originarias: Cooperación, Competición, Individualismo y Afiliación que explicaron un $66.43 \%$ de la varianza y presentaron valores elevados de consistencia interna. Por otro lado, los resultados indican una equivalencia factorial estricta. Los profesores con más años de experiencia prefieren escenarios de trabajo más individualistas y cooperativos que aquellos con menor experiencia y los hombres tienen preferencias más individualistas y competitivas, mientras que las mujeres tienen mayor orientación cooperativa.
\end{abstract}

Palabras clave: Interacción social; Medición de la invarianza; Análisis factorial confirmatorio; Educación física; Profesorado.

\section{Introducción}

Todos los movimientos educativos y las reformas que se han realizado en las últimas décadas han promocionado la relación entre el personal docente, destacando el papel de las actividades colaborativas y cómo éstas pueden contribuir a la mejora de la actuación docente (Morrison, Wakefield, Walker y Solberg, 1994).

En el ámbito de la formación del profesorado de educación física son numerosos los estudios y experiencias que se han llevado y se vienen llevando a cabo hasta la fecha (Castro, Gómez y Macazaga, 2014; Flores, Prat i Grau y Soler Prat, 2014; García, 1997; González y Barba, 2014). Los estudios realizados para analizar su integración en su entorno laboral han estado referidos al análisis de su satisfacción laboral (Anaya y Suárez, 2010) o a la evaluación del desempeño docente (Cárdenas, Méndez y González, 2013). Asimismo, estudios recientes han destacado la importancia de mejorar las competencias docentes para el control del grupo y la resolución de problemas en el entorno laboral (Tani, 2013). En esta línea Yaylaci (2011) destaca la importancia de los valores personales, el conocimiento de los estudiantes, la gestión de las conductas en clase y el control del proceso de aprendizaje

* Dirección para correspondencia [Correspondence address]: Maria-Isabel Barriopedro. Departamento de Ciencias Sociales de la Actividad Física, del Deporte y del Ocio. Facultad de Ciencias de la Actividad Física y Deporte-INEF. Avda. Martín Fierro s/n. Universidad Politécnica de Madrid. 28040 Madrid (España).

E-mail: misabel.barriopedro@upm.es
Title: The physical education teachers' participative preferences in their professional activity.

Abstract: Nowadays the collaborative learning environments have been promoted by the physical education teachers as the best way for decreasing teaching problems independently of teachers' personal achievements. The aim of the present study was to develop a scale for physical education teachers about social interactions preferences in the workplace environment. Based on the scale GR developed by Ruiz et al. (2010), the multisample confirmatory factor analysis was used to assess the equivalence measure between years of experience and gender. The participants were 1413 Spanish physical education teachers. The final scale was composed of the 4 initial dimensions: cooperation, competition, individualization, and affiliation. The four dimensions accounted for $66.43 \%$ of the total variance, and showed high values of internal consistency. Also, the results pointed out a strict factorial invariance. Finally, the results showed that the more experienced teachers prefer individualized and competitive workplace environments than the less experienced ones, and on the other hand, men's teachers were more individualistic and competitive compared with women's teachers whom prefer cooperative orientations in the workplace environment.

Key words: social interaction; invariance measurement; confirmatory factorial analysis; physical education; teachers.

como los aspectos prioritarios de la labor profesional y en consecuencia de la formación del profesorado Sin embargo, tal y como establece este autor (Yaylaci, 2011) el profesorado está afectado por factores internos (sus propias percepciones, estudiantes y padres/ madres) y externos (compañeros de trabajo, directores y jefes de estudio de los centros, y las disposiciones legales en materia educativa) que condicionan su labor profesional, y por tanto, se deben fomentar y potenciar herramientas formativas y de evaluación, para mejorar de manera positiva la motivación del profesorado y de este modo la del grupo clase.

En este sentido, un estudio reciente de Thorp (2014) destacó que uno de los objetivos prioritarios del profesorado de educación física es motivar a los estudiantes para la práctica, sin embargo, tres factores condicionan la motivación a participar en las clases de educación física: 1) el género: los chicos y las chicas están motivados de diferente modo ante las prácticas deportivas (p.e., los chicos hacia tareas competitivas, individuales o deportes de equipo como el fútbol o baloncesto; mientras que las chicas hacia actividades cooperativas y saludables); 2) la imagen corporal: la actividad física debe alcanzar más que el estereotipo dominante de apariencia física, por ello la creación de ambientes de trabajo cooperativo y motivante ayudará a mejorar la adhesión a la práctica deportiva; y 3) el disfrute: el cual está influenciado por la figura del profesor, las características del estudiante, las actividades y el contenido de las clases, y el ambiente de aprendizaje; de acuerdo con estos aspectos la influencia del profesorado es muy elevada en las instrucciones, la evaluación, la motivación 
y el control del grupo de clase para aumentar el disfrute. Thorp (2014) considera que el profesorado de educación física debe considerar estos 3 factores para poder crear ambientes de aprendizaje de disfrute y cooperativos.

De acuerdo con las ideas previas, el zeitgeist pedagógico en el ámbito de la educación física ha provocado que en la mayoría de los casos se hayan promocionado los entornos colaborativos como la mejor solución a los problemas que la actividad docente puede presentar (Barrett, 2005; Dyson, 2001, 2002; Dyson y Grineski, 2001; Orlick, 1978a, 1978b, 1988, 2006; Polvi y Telama, 2000) más allá de las preferencias personales de los profesores, preferencias de las cuales existe una escasa investigación hasta la fecha, desconociendo si los profesores son preferentemente cooperativos o competitivos en sus entornos laborales, o por el contrario son más individualistas o afiliativos. La idea predominante ha sido destacar las bondades de un entorno cooperativo frente al competitivo o el individualista, ya que con ello se conseguirían mejores resultados en las clases (Dyson y Strachan, 2000).

Estas ideas han calado en la propia formación del profesorado ya que una parte importante de la investigación se ha basado en analizar los entornos de aprendizaje cooperativocompetitivo que los profesores crean en clase, a partir de las propuestas de los hermanos Johnson (Johnson, 1981; Johnson, Johnson y Holubec, 1994; Johnson, Maruyama, Johnson, Nelson y Skon, 1981), quienes destacaron el efecto favorable de los entornos colaborativos en el aprendizaje. Sin embargo han sido escasos los estudios que hayan analizado en escolares o profesores sus preferencias participativas en entornos de aprendizaje y menos en entornos laborales.

En el 2010 Ruiz, Graupera, Moreno y Rico acometieron la labor de explorar las preferencias de interacción de los escolares de secundaria para lo cual desarrollaron la Escala GR para el análisis de las preferencias sociales de interacción en educación física. Esta escala posee una estructura tetrafactorial ya que a las conocidas dimensiones de Cooperación, Competición e Individualismo añadieron una más denominada Afiliación. Pero ¿qué significan cada una de ellas y qué pueden significar cuando de lo que se trata es de conocer las preferencias de interacción social de los profesores de educación física en sus entornos de trabajo y por qué puede ser relevante conocerlas?

Johnson (1981) y Johnson, Johnson y Holubec (1994) definieron las tres primeras estructuras de interacción de la siguiente forma:

- Cooperativa. Cuando los objetivos de los participantes están estrechamente interconectados y un miembro consigue su objetivo cuando los otros miembros del grupo también lo consiguen. Lo que supondría que en un departamento de educación física de un centro escolar todos los profesores y profesoras del mismo trabajan de manera estrecha para conseguir los objetivos que el centro ha planteado y que la materia reclama en cada curso.

- Competitiva. Cuando los resultados no están interconectados. Si un profesor consigue su objetivo puede ser que otros profesores del departamento no los consigan. Cada profesor y profesora compite con el resto de los componentes del departamento en la búsqueda de su mayor logro personal. Esto supondría que cada profesor o profesora trabajaría de manera concreta con sus grupos, tomaría a sus compañeros como oponentes a los que superar, buscando ser reconocido por encima de ellos.

- Individualista. En este contexto no existe relación entre los objetivos que los componentes tratan de obtener. El que uno de los componentes del grupo alcance su objetivo no afecta ni impide que otros componentes del grupo puedan obtenerlos. Esta organización social se caracteriza porque sus miembros no están preocupados ni interesados por el progreso de los demás. Implica que el profesor se aísla del resto de compañeros del departamento, lleva su propia línea de trabajo, se despreocupa por la trayectoria docente de sus colegas y solo se preocupa de sí mismo.

A estas estructuras clásicas y con relación al alumnado, Ruiz et al. (2010) añadieron una más denominada Afiliación en la que, a diferencia de la dimensión cooperativa, no existe una relación entre iguales. La persona que predomina en esta dimensión no desea tomar decisiones, prefiere pertenecer a un grupo donde las reglas estén establecidas y otros sean los que decidan por él, constituyéndose el grupo en un entorno de seguridad.

En este sentido, parece adecuado analizar y conocer cuáles son las preferencias que manifiestan los propios profesores, aquellos que son responsables de generar estructuras de aprendizaje y los contextos de práctica. Desde un punto de vista tradicional la formación del profesorado ha insistido en llevar a cabo seminarios y programas de cooperación con otros profesores de educación física, asumiendo que las preferencias participativas del profesorado son de corte cooperativo, ¿pero es así?, los estudios al respecto son escasos y como Tani (2013) sugiere, se hace necesario incorporar a dichos programas los avances científicos que mejoren la ensenanza. Conocer las preferencias participativas del profesorado de educación física permitiría favorecer la propia formación de los docentes y se asociaría a la dimensión cooperativa del modelo de formación del profesorado otras dimensiones también presentes en las aulas y en los gimnasios que podrían cambiar en función del género y la experiencia profesional de los profesores, lo cual redundaría en un aumento de su motivación al percibir que sus preferencias son consideradas en su propia formación (Tani, 2013; Thorp, 2014; Yailaci, 2011).

Es por ello que el objetivo de este estudio haya sido la validación de una escala que permita conocer las preferencias sociales de interacción en el entorno laboral de los profesores de educación física. Asimismo resultó de interés analizar estas preferencias en función del género y su experiencia docente. 


\section{Método}

\section{Participantes}

En este estudio participaron 1413 profesores de educación física españoles repartidos por toda la geografía española, de edades comprendidas entre los 20 y los 65 años $(M=$ 33.4; $D T=10.2$ años), y de los que 925 eran hombres $(M=$ 33.4; $D T=10.1$ años) y 488 mujeres $(M=33.5 ; D T=10.2$ años). El $74.9 \%$ de la muestra eran profesores con una experiencia menor de 15 años y una experiencia docente promedio de $4.6 \pm 4.2$ años y el $25.1 \%$ eran profesores con más de 15 años de experiencia docente, con una experiencia promedio de $22.4 \pm 5.8$ años. Del conjunto de la muestra, 294 eran profesores en Educación Primaria, 612 en Educación Secundaria y 507 en Bachillerato. En cuanto a la formación académica 311 eran Maestros Especialistas en Educación Física, 701 Graduados o Licenciados en Ciencias de la Actividad Física y 401 estudiantes de último curso en Ciencias de la Actividad Física y Deportes. En esta muestra hubo participantes de todas Comunidades Autónomas, incluidas las Ciudades Autónomas de Ceuta y Melilla, de centros públicos, privados y concertados.

\section{Instrumentos}

Para el desarrollo de la escala de preferencias de interacción social en el entorno laboral de los profesores de educación física se partió de la Escala GR desarrollada por Ruiz et al. (2010). Para que pudiese responder al criterio básico de que los ítems estuvieran referidos al Seminario o el Departamento de Educación Física del Centro, se adaptaron los ítems, de tal forma que si el ítem $1^{\circ}$ en la escala original se expresaba como " Me gusta hacer las cosas mejor que los demás", en esta nueva escala quedó como "Me gusta hacer las cosas mejor que los demás profesores/as de mi seminario o departamento". Estos pequeños cambios fueron presentados a 4 profesores de educación física en ejercicio quienes confirmaron que eran claros y no presentaban problemas de comprensión.

La escala objeto de estudio estuvo constituida por 28 ítems con un sistema de respuesta tipo Likert de 1 (totalmente en desacuerdo) a 9 (totalmente de acuerdo). Ejemplos de las cuatro dimensiones son: (a) dimensión competitiva: "Me gusta hacer las cosas mejor que los/las demás profesores/as de mi seminario o departamento", (b) Dimensión Cooperativa: "Me gusta decir y hacer cosas que ayuden a los demás colegas del seminario o departamento", (c) Dimensión Afiliativa: "Trabajo en grupo para que deseen estar conmigo.", y (d) Dimensión Individualista: "Me gusta trabajar a mi manera, sin tener que depender de lo que hagan los demás profesores/as". En la Tabla 1 se presentan los ítems de la versión inicial de la escala. Asimismo se aplicó una adaptación al profesorado del Cuestionario de Orientación al Ego y a la Tarea (TEOSQ) (Balaguer, Castillo y Tomás, 1996; Peiró, 1999) y que fue utilizado para valorar la validez de criterio de la escala de preferencias.
Tabla 1. Ítems de la versión inicial de la escala.

1. Me gusta hacer las cosas mejor que los demás profesores/as de mi seminario o departamento.

2. Me gusta decir y hacer cosas que ayuden a los demás colegas del seminario o departamento.

3. Trabajo en grupo para que deseen estar conmigo.

4. Me gusta trabajar a mi manera, sin tener que depender de lo que hagan los demás. profesores/as.

5. Me gusta ser capaz de hacer las cosas mejor que mis compañeros y compañeras del departamento o seminario.

6. Me gusta participar en trabajos de grupo en el departamento o seminario.

7. Necesito participar en grupo para sentirme bien.

8. Mi mejor manera de trabajar bien es trabajar solo/a.

9. Intento ser el/la mejor profesor/a del colegio.

10. Creo que el trabajo en grupo es necesario para todos.

11. Me gustan las actividades de grupo porque siempre sé lo que tengo que hacer.

12. Deseo hacer tareas individuales en el departamento, para poder trabajar solo/a.

13. Me gusta ser más eficiente que los demás colegas.

14. Me encantan las tareas de equipo.

15. Me gustan las actividades docentes en grupo porque se notan menos los fallos.

16. Me siento mejor cuando trabajo solo/a en las actividades del departamento.

17. Me gusta que me digan que lo he hecho mejor que los demás profesores/as de mi departamento o seminario.

18. Sólo me siento bien cuando trabajo en equipo.

19. Me encantan las tareas individuales.

20. Trabajo más duro que los demás cuando intento superar a mis compañeros y compañeras del departamento o seminario.

21. Deseo trabajar con los demás colegas, aunque sea en tareas muy aburridas.

22. Las cosas me salen mejor cuando colaboro con los demás profesores/as que cuando estoy solo/a.

23. La mejor manera de aprender en mi profesión es trabajando solo/a.

24. Trabajo más duro en algo, cuando veo que los demás profesores del departamento o seminario lo hacen mejor que yo.

25. Me gusta realizar trabajos en grupo, aunque sean más difíciles.

26. Cuando trabajo solo/a es cuando consigo mejor mis objetivos como profesor/a.

27. Creo que intentar estar siempre entre los más eficaces, me ayuda mucho a aprender y a mejorar.

28. Lo más gratificante de ser profesor es el trabajo individual y la reflexión personal.

\section{Procedimiento}

Se tomó contacto con los profesores y profesoras en persona, por correo postal o por correo electrónico. Todos fueron informados de la naturaleza del estudio, de su anonimato y de la privacidad que reclaman este tipo de estudios dando su consentimiento voluntario para participar en él. Los docentes participantes cumplimentaron la escala, lo cual no les llevó más de 15 minutos, no presentándose problemas de comprensión de los ítems dignos de ser considerados. 


\section{Análisis de Datos}

Para analizar la estructura factorial de la escala se seleccionaron al azar la mitad de los cuestionarios (707) y se aplicó un Análisis Factorial Exploratorio (AFE). Se utilizó el método de ejes principales para la extracción de factores y rotación oblimin directo, adecuado cuando se asume correlación entre los factores analizados (Worthington y Whittaker, 2006). La adecuación de la matriz para realizar el AFE fue testada mediante el test Kaiser-Meyer-Olkin (KMO) y la Prueba de Esfericidad de Bartlett. Para eliminar ítems se siguieron los siguientes criterios: comunalidades inferiores a 0.40 , peso factorial menor de 0.40 , pesos superiores a 0.25 en más de un factor y aquellos en los que la diferencia de pesos entre dos factores fuera inferior a 0.15 (Worthington y Whittaker, 2006). Para las dimensiones obtenidas de este análisis se realizó un análisis de la fiabilidad mediante el coeficiente Alpha.

La estructura factorial obtenida mediante el AFE fue sometida a un Análisis Factorial Confirmatorio (AFC) con la otra mitad de la muestra (706 sujetos). El método de Máxima Verosimilitud (ML) fue utilizado para estimar los parámetros, tras comprobar que tanto los índices de Asimetría como los de Apuntamiento de los ítems no presentaban valores elevados (Asimetría y Apuntamiento menor que 2 en valor absoluto). Para evaluar la bondad de ajuste de los datos al modelo propuesto se utilizaron los siguientes índices: Raíz del Error Cuadrático Medio de Aproximación (RMSEA) y su intervalo de confianza al 90\%, Índice de Ajuste Comparado de Bentler (CFI) y el valor relativo de Chi Cuadrado $\left(\chi^{2} / \mathrm{gl}\right)$.

En la literatura, valores de RMSEA $\leq .05$ o .08 se consideran como indicadores de un buen ajuste o un ajuste aceptable, respectivamente; valores de CFI $\geq .90$ han sido aceptados como indicadores de un buen ajuste; por último valores relativos de Chi Cuadrado entre 2 y 5 se consideran como indicadores de ajuste aceptable. Para comprobar si la estructura factorial era igual en profesores expertos y novatos y en hombres y mujeres se realizaron AFC multigrupo.

La invarianza factorial se evaluó de forma progresiva (Byrne, 2008; Elosua, 2005): invarianza configural (el patrón de cargas factoriales es el mismo); invarianza métrica o débil (además del patrón de cargas, los pesos factoriales son iguales); invarianza escalar o fuerte (además de invarianza métrica supone que los interceptos son iguales) e invarianza estricta (además de la invarianza escalar supone varianzas iguales para los errores).

Por último, se incluyó un modelo de invarianza que además de suponer la invarianza estricta incluía la invarianza de las covarianzas entre factores. Para comparar el ajuste de los modelos se utilizó el Criterio de Información de Akaike (AIC) y la comparación de los valores del CFI. Si la diferencia entre los valores CFI de dos modelos anidados es superior a .01 a favor del modelo menos restrictivo deberá rechazarse el modelo con más restricciones (Chenung y Rensvold, 2002). Por último se realizó un MANOVA de dos factores para analizar las diferencias en las subescalas en función de la experiencia docente y el sexo. Las comparaciones múltiples se llevaron a cabo con la prueba de Bonferroni y se calculó eta cuadrado parcial $\left(\eta^{2}\right)$ para cuantificar el tamaño del efecto. El nivel de riesgo se fijó en .05. Los análisis fueron realizados mediante el programa PASW 20.0 y el programa AMOS 21.0.

\section{Resultados}

\section{Dimensionalidad de la escala}

En la Tabla 1 se presentaron los ítems que formaron la versión inicial del cuestionario. Tras someter los 28 ítems a un análisis factorial exploratorio, 5 de ellos (ítems 19, 21, 23, $24,26)$ fueron eliminados al presentar comunalidades inferiores a .40 , y 4 de ellos se eliminaron al presentar cargas factoriales en más de un factor (ítems 7, 20, 22 y 27). El índice KMO (.91) y la prueba de esfericidad de Barlett $\left(\chi^{2}(171)=\right.$ 141.64; $p<.001)$, tras someter a un análisis factorial exploratorio los 19 ítems restantes, pusieron de manifiesto la adecuación de la matriz para el análisis. La solución factorial obtenida estuvo formada por 4 factores que explicaron el $66.43 \%$ de la varianza (Tabla 2). El primer factor (Dimensión Competitiva) explicó el $31.78 \%$ de la varianza y estuvo constituido por 5 ítems $(1,5,13,9,17)$. El segundo factor (Dimensión Cooperativa), estuvo integrado por 5 ítems $(6,10,2$, $14,25)$ y explicó el $19.82 \%$ de la varianza. El tercer factor (Dimensión Individualista), lo formaron 5 ítems (16, 8, 12, 4, 28) explicando el $8.45 \%$ de la varianza y por último, el cuarto factor (Dimensión Afiliativa) estuvo integrado por 4 ítems $(18,15,3,11)$ y explicó el $6.40 \%$ de la varianza. Las correlaciones entre los factores se presentan en la Tabla 3.

\section{Fiabilidad}

El coeficiente alfa fue de .90 para la subescala Competitiva, .92 para la Cooperativa, .87 para la subescala Individualista y .71 para la Afiliativa. Todas las subescalas presentaron coeficientes superiores a .70 que indican una consistencia aceptable en el ámbito de la investigación psicológica (Nunnally, 1978).

\section{Análisis Factorial Confirmatorio e Invarianza Facto- rial}

Con objeto de poner a prueba la estructura factorial encontrada en el análisis anterior se realizó un análisis factorial confirmatorio con la otra mitad de la muestra. Los resultados del análisis indicaron un buen ajuste del modelo propuesto $\left(\right.$ RMSEA $\left.=.046(.040-.052) ; \mathrm{CFI}=.96 ; \chi^{2}(139)=347.7\right)$. Sólo mostraron una correlación significativa la subescala Individualista con la Competitiva $(\mathrm{r}=.44 ; p<.001)$ y la Cooperativa $(\mathrm{r}=-.58 ; p<.001)$ y la subescala Afiliativa con la Competitiva $(\mathrm{r}=.47 ; p<.001)$ y con la Cooperativa $(\mathrm{r}=.51$; $p<.001)$. 
Tabla 2. Solución factorial de primer orden.

\begin{tabular}{|c|c|c|c|c|}
\hline Ítem & Competitiva & Cooperativa & Individualista & Afiliativa \\
\hline $\begin{array}{l}\text { 1. Me gusta hacer las cosas mejor que los demás profesores/as de mi departamento o } \\
\text { seminario. }\end{array}$ & .916 & & & \\
\hline $\begin{array}{l}\text { 5. Me gusta ser capaz de hacer las cosas mejor que mis compañeros y compañeras del } \\
\text { departamento o seminario. }\end{array}$ & .906 & & & \\
\hline 13.Me gusta ser más eficiente que los demás colegas. & .853 & & & \\
\hline 9. Intento ser el/la mejor profesor/a del colegio. & .747 & & & \\
\hline $\begin{array}{l}\text { 17. Me gusta que me digan que lo he hecho mejor que los demás profesores/as de mi } \\
\text { departamento o seminario. }\end{array}$ & .627 & & & \\
\hline 6. Me gusta participar en trabajos de grupo en el departamento o seminario. & & .895 & & \\
\hline 10.Creo que el trabajo en grupo es necesario para todos. & & .879 & & \\
\hline $\begin{array}{l}\text { 2. Me gusta decir y hacer cosas que ayuden a los demás colegas del seminario o depar- } \\
\text { tamento. }\end{array}$ & & .828 & & \\
\hline 14.Me encantan las tareas de equipo & & .749 & & \\
\hline 25.Me gusta realizar trabajos en grupo, aunque sean más difíciles. & & .741 & & \\
\hline 8. Mi mejor manera de trabajar bien es trabajar solo/a. & & & .838 & \\
\hline 16.Me siento mejor cuando trabajo solo/a en las actividades del departamento. & & & .815 & \\
\hline 12.Deseo hacer tareas individuales en el departamento, para poder trabajar solo/a. & & & .746 & \\
\hline $\begin{array}{l}\text { 4. Me gusta trabajar a mi manera, sin tener que depender de lo que hagan los demás } \\
\text { profesores/as. }\end{array}$ & & & .735 & \\
\hline 28.Lo más gratificante de ser profesor es el trabajo individual y la reflexión personal. & & & .705 & \\
\hline 18.Sólo me siento bien cuando trabajo en equipo. & & & & .746 \\
\hline 15.Me gustan las actividades docentes en grupo porque se notan menos los fallos. & & & & .731 \\
\hline 11.Me gustan las actividades de grupo porque siempre sé lo que tengo que hacer. & & & & .592 \\
\hline 3. Trabajo en grupo para que deseen estar conmigo. & & & & .560 \\
\hline$\%$ Varianza Explicada & 31.78 & 19.82 & 8.45 & 6.40 \\
\hline$\alpha$ & .90 & .92 & .87 & .71 \\
\hline
\end{tabular}

Tabla 3. Correlaciones entre los factores de primer orden.

\begin{tabular}{|c|c|c|c|c|}
\hline & Competitiva & Cooperativa & Individualista & Afiliativa \\
\hline Competitiva & & $.163^{*}$ & $.465^{*}$ & $.380^{*}$ \\
\hline Cooperativa & & & -.011 & $.270 *$ \\
\hline Individualista & & & & $.155^{*}$ \\
\hline
\end{tabular}

Los índices de ajuste obtenidos (Tabla 4) permiten aceptar la equivalencia del patrón de cargas factoriales (invarianza configural) entre docentes novatos y expertos. Aunque el valor de Chi-cuadrado es significativo el resto de los índices avalan esta conclusión (RMSEA $=.036$ y CFI $=.956$ ). Cuando al modelo se le añaden restricciones sobre los pesos factoriales (invarianza métrica) obtenemos un buen ajuste del modelo (RMSEA $=.035$ y CFI = .956). Por otra parte, el criterio de información de Akaike (AIC $=799.3$ ) y el índice comparativo de Bentler no sufrieron modificaciones apreciables. Estos resultados permiten aceptar la invarianza métrica. Cuando se añade al modelo la restricción de interceptos (invarianza escalar), tanto el índice de ajuste de Bentler (CFI = .957) como la raíz del error cuadrático medio de aproximación $($ RMSEA $=.034$ ) aportan valores de ajuste aceptables, la reducción del CFI fue menor de .01 con respecto al modelo de invarianza métrica y el AIC (777.9) no sufrió una variación notable, datos que nos llevan a aceptar la invarianza escalar.

El modelo que supone invarianza estricta, también mostró índices de ajuste aceptables, no apreciándose una modificación importante del CFI (se reduce en .008) ni del AIC con respecto al modelo escalar, por lo que puede ser aceptada la invarianza estricta. Por último, el modelo que supone invarianza estricta más la invarianza de las covarianzas entre los factores muestra índices que indican un buen ajuste (RMSEA $=.035$ y CFI $=.948)$ y además no se observa un incremento importante de AIC (794.8) con respecto al modelo de invarianza estricta y la diferencia entre los Índices de Bentler entre estos dos modelos es inferior a .01.

Los índices de ajuste obtenidos (Tabla 5) permiten aceptar el modelo de invarianza estricta más la invarianza de las covarianzas entre factores entre hombres y mujeres, ya que para todos los modelos se obtienen valores de RMSEA adecuados, en ningún caso la diferencia en los valores de CFI asociados a los modelos jerárquicos consecutivos supera el valor .01 y no se observan incrementos importantes del AIC.

En la Figura 1 se presentan los pesos factoriales estandarizados, las correlaciones múltiples al cuadrado entre los factores y los ítems, y las correlaciones entre los factores y entre los errores. Los ítems fueron buenos indicadores de sus factores latentes respectivos, con cargas factoriales entre $0.65 \mathrm{y}$ 0.90 para la dimensión Competitiva, entre .34 y .82 para la dimensión Cooperativa, entre .52 y .83 para la Individualista y con cargas entre .53 y .69 para la dimensión Afiliativa. Todos los pesos resultaron estadísticamente significativos $(p<$ .001). El modelo estimó una correlación significativa entre la subescala de Orientación Individualista con la subescala Competitiva $(r=.44 ; p<.001)$ y con la Cooperativa $(r=$ $.58 ; p<.001)$ y entre la subescala de Orientación Afiliativa con la Competitiva $(r=.47 ; p<.001)$ y con la Cooperativa $(r$ $=.51 ; p<.001)$. 
Tabla 4. Índices de bondad de ajuste de los modelos de invarianza por experiencia docente.

\begin{tabular}{lcccrcc}
\hline Invarianza & $\chi^{2}$ & $\mathrm{gl}$ & $\chi^{2} / \mathrm{gl}$ & RMSEA (IC al 90\%) & CFI & AIC \\
\hline Configural & 533.9 & 278 & 1.92 & $.036(.031-.041)$ & .956 & 813.9 \\
Métrica & 549.3 & 293 & 1.89 & $.035(.031-.040)$ & .956 & 799.3 \\
Escalar & 563.9 & 312 & 1.81 & $.034(.029-.038)$ & .957 & 777.9 \\
Estricta & 638.8 & 340 & 1.88 & $.035(.031-.039)$ & .949 & 794.8 \\
Estricta +Covarianza Factores & 569.9 & 316 & 1.8 & $.035(.031-.039)$ & .948 & 794.8 \\
\hline
\end{tabular}

Tabla 5. Índices de bondad de ajuste de los modelos de invarianza por sexo.

\begin{tabular}{|c|c|c|c|c|c|c|}
\hline Invarianza & $\chi^{2}$ & $\mathrm{gl}$ & $\chi^{2} / g 1$ & RMSEA (IC al 90\%) & CFI & $\mathrm{AIC}$ \\
\hline$\overline{\text { Configural }}$ & 527.2 & 278 & 1.90 & $.036(.031-.040)$ & .957 & 807.2 \\
\hline Métrica & 559.8 & 293 & 1.91 & $.036(.031-.040)$ & .954 & 809.8 \\
\hline Escalar & 627.5 & 312 & 2.01 & $.038(.033-.042)$ & .946 & 839.5 \\
\hline Estricta & 694.5 & 340 & 2.04 & $.038(.034-.042)$ & .940 & 850.5 \\
\hline Estricta + Covarianza Factores & 702.7 & 348 & 2.02 & $.038(.034-.042)$ & .940 & 842.7 \\
\hline
\end{tabular}

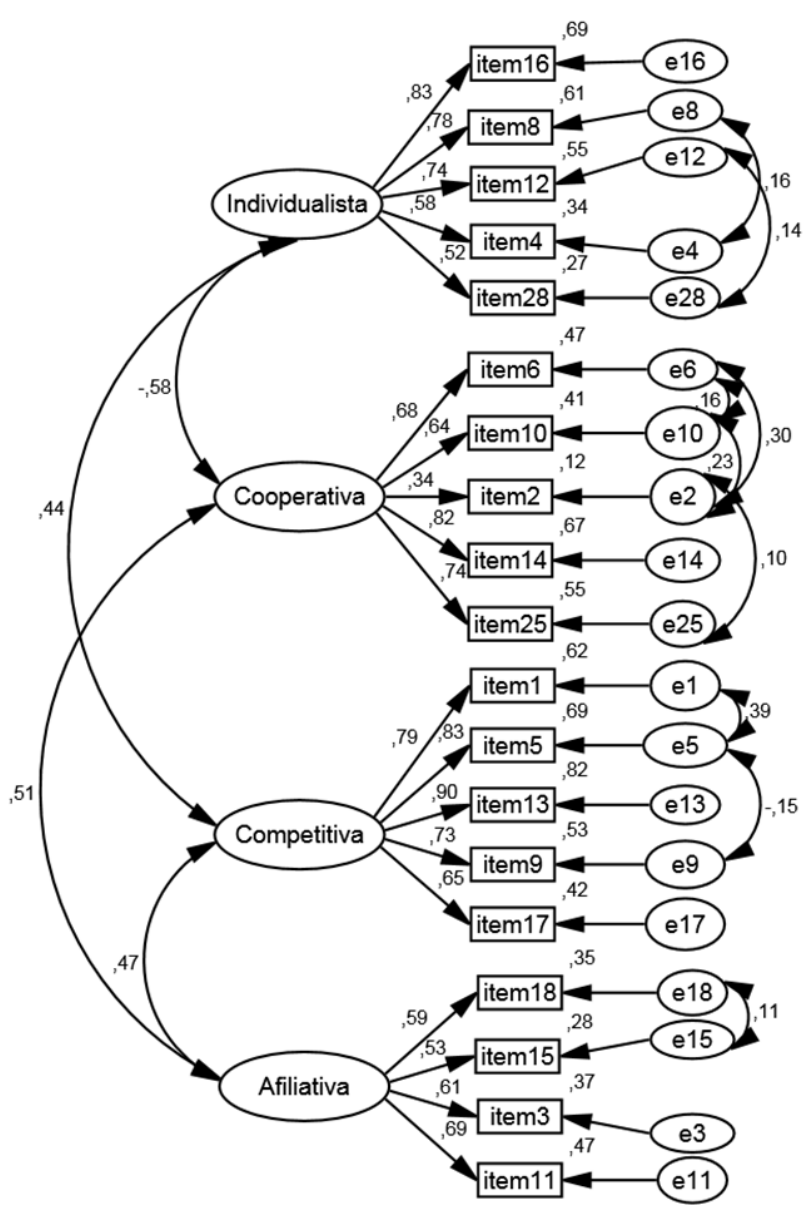

Figura 1. Modelo estructural para la escala de barreras. Pesos estandarizados, correlaciones múltiples al cuadrado y correlaciones entre factores y términos error.

\section{Validez aparente}

La validez aparente hace referencia a la impresión que causa en los participantes el instrumento. Suponen juicios sobre el instrumento una vez que ya ha sido construido. Para evaluar este tipo de validez se preguntó a los participantes una vez cumplimentado el test, en qué grado les había gustado el cuestionario, para lo cual se les ofrecía una escala ordinal de 1 a 10 indicando el significado de ambos extremos. La valoración fue de 9.10 sobre 10 indicando una buena validez aparente.

\section{Validez criterial}

La validez criterial se refiere al grado en que un constructo se comporta como debería en un sistema de constructos relacionados. En el caso de esta escala de preferencias sociales se analizó el grado de relación con otros constructos evaluados en el ámbito de la Psicología del Deporte. El constructo considerado fue la orientación motivacional de los profesores participantes en el estudio evaluado mediante Cuestionario de Orientación al Ego y a la Tarea (TEOSQ) (Balaguer, Castillo y Tomás, 1996; Peiró 1999). Los resultados mostraron que la mayoría de los coeficientes de correlación fueron significativos $(p<.001)$. Las relaciones fueron bajas pero consistentes (Tabla 6 ).

Tabla 6. Correlaciones entre los factores de primer orden.

\begin{tabular}{lcc}
\hline & Clima EGO & Clima TAREA \\
\hline Competitiva & $.23^{*}$ & $.07^{*}$ \\
Cooperativa & $-.15^{*}$ & $.20^{*}$ \\
Individualista & $.13^{*}$ & - \\
Afiliativa & $.17^{*}$ & - \\
\hline$* p<.001$ & &
\end{tabular}

Preferencias participativas, experiencia docente y género

El análisis de las puntuaciones obtenidas en las cuatro dimensiones de la escala (Tabla 7) arrojó efecto multivariado de la experiencia docente $(F(4,1406)=12.29 ; p<.001)$, del sexo $(F(4,1406)=6.84 ; p<.001)$ pero no de la interacción $(F(4,1406)=1.36 ; p=.245)$. Los contrastes univariados pusieron de manifiesto que los profesores con más de 15 años de experiencia (expertos) presentaron puntuaciones superiores a los profesores con menos de 15 años de experiencia en 
las subescalas de Orientación Individualista $(F(1,1409)=$ $\left.6.08 ; p=.014 ; \eta^{2}=.004\right)$ y Cooperativa $(F(1,1409)=31.57 ; p$ $\left.<.001 ; \eta^{2}=.022\right)$, no observándose diferencias en las subescalas de Orientación Competitiva $(F(1,1409)=0.01 ; p=.997)$ y Afiliativa $(F(1,1409)=0.74 ; p=.388)$. Los hombres presentaron puntuaciones superiores en las subescalas de Orientación Individualista $\left(F(1,1409)=10.98 ; \mathrm{p}=.001 ; \eta^{2}=.008\right)$ y Competitiva $\left(F(1,1409)=17.77 ; p<.001 ; \eta^{2}=.012\right)$ mientras que las mujeres presentaron puntuaciones superiores a los hombres en la subescala Cooperativa $(F(1,1409)=3.93$; $p$ $\left.=.048 ; \eta^{2}=.003\right)$. No se observaron diferencias significativas en función del sexo en la subescala de Orientación Afiliativa $(F(1,1409)=3.29 ; p=.070)$.

Tabla 7. Análisis descriptivo de las escalas en función de la experiencia docente y del sexo (media \pm desviación típica).

\begin{tabular}{lcccc}
\hline & \multicolumn{2}{c}{ Novatos } & \multicolumn{2}{c}{ Expertos } \\
\cline { 2 - 5 } & Hombres & Mujeres & Hombres & Mujeres \\
\hline Individualista & $19.2 \pm 8.8$ & $16.7 \pm 7.9$ & $19.9 \pm 9.3$ & $18.7 \pm 9.0$ \\
Cooperativa & $31.7 \pm 8.9$ & $31.9 \pm 9.9$ & $34.0 \pm 7.8$ & $36.0 \pm 7.5$ \\
Competitiva & $22.0 \pm 10.4$ & $19.1 \pm 9.8$ & $21.8 \pm 10.3$ & $19.2 \pm 10.9$ \\
Afiliativa & $15.6 \pm 6.5$ & $14.3 \pm 6.6$ & $15.4 \pm 6.5$ & $15.2 \pm 6.7$ \\
\hline
\end{tabular}

\section{Discusión}

El propósito fundamental de este estudio fue desarrollar una escala de preferencias de interacción social en el entorno laboral para los profesores de educación física así como evaluar la equivalencia de medida entre profesores con diferentes años de experiencia docente y género, utilizando el análisis factorial confirmatorio multimuestra. Esto ha permitido conocer las preferencias participativas de los profesores, los mismos que siguiendo las tendencias pedagógicas actuales promocionan entornos colaborativos.

Para el desarrollo de la escala se partió de la Escala GR de Ruiz et al. (2010) y una muestra de más de un millar de profesores y profesoras de educación física. Se aplicó un AFE a la mitad de la muestra seleccionada al azar. Los 19 ítems que constituyen la escala final se agruparon en las 4 cuatro dimensiones originarias: Cooperación, Competición, Individualismo y Afiliación que explicaron un $66.43 \%$ de la varianza, estos resultados se encuentran dentro los valores entre el $60 \%$ y $70 \%$ de la varianza explicada en la validación de cuestionarios previos del ámbito educativo en profesorado y alumnado de educación física (Fernández, Sánchez, y Salinero, 2008; Ruiz et al., 2010; Sánchez-Oliva, SánchezMiguel, Miguel-Leo, Amado, y García-Calvo, 2013)

La escala ha resultado fiable en sus diferentes dimensiones mostrando una elevada consistencia interna. El análisis factorial confirmatorio llevado a cabo con la otra mitad de la muestra presentó un ajuste satisfactorio del modelo obtenido mediante el análisis factorial exploratorio. El estudio progresivo de la invarianza factorial entre profesores novatos y expertos por un lado, y hombres y mujeres por otro, mostró un ajuste adecuado del modelo que supone una invarianza estricta así como el que supone que, además de esta invarianza estricta, las relaciones entre los factores eran equivalentes en los distintos grupos.

Algunos autores (Coenders, Batista y Saris, 2005) sostienen la necesidad de comprobar la igualdad de interceptos para poder comparar las medias de los factores entre grupos, aunque sería suficiente con una invarianza escalar parcial (bastaría que se cumpliese para una parte de los ítems de cada dimensión). En este sentido, este estudio ha permitido constatar que lo profesores más experimentados prefieren escenarios de trabajo en los que se alterne la actuación individual como la relación de cooperación con los demás colegas del seminario o departamento, si bien la preferencia cooperativa destaca en ambos grupos seguida por la competitiva.

Estos resultados están en consonancia con los resultados de Caussidier et al. (2011) sobre el profesorado de educación física en 4 países del Mediterráneo (Francia, Líbano, Túnez y Marruecos) que muestra que los factores socioculturales y personales son los que más influyen en el desarrollo de las clases de educación física, principalmente la experiencia previa, siendo el profesorado con más años de trabajo más capaz de planificar y preparar de manera coordinada sus programaciones, y por tanto favorecer el aprendizaje y el clima de clase. Siguiendo esta línea, el estudio de Yaylaci (2011) establece en sus conclusiones principales que el trabajo individualizado de formación continua y el trabajo cooperativo entre el profesorado debe ser una prioridad para las universidades así como para las instituciones educativas públicas, consiguiendo con ello un aumento de la motivación del profesorado, mejorando la calidad de las clases y del clima de aprendizaje.

Thorp (2014) establece que por un lado el profesorado debe mejorar a nivel individual las competencias de conocimiento específico de la materia y su aplicación en su contexto específico, y por otro el trabajo coordinado de planificación de las programaciones y estándares necesarios en función de la edad y del contexto de trabajo. Por lo tanto, los resultados obtenidos son explicativos de la realidad del profesorado y de la necesidad formativa del mismo que se centra a nivel individual y colectivo en cooperación con sus compañeros de profesión.

Por último, los resultados en función del género mostraron que los hombres tenían preferencias más individualistas y competitivas, mientras que las mujeres tenían mayor orientación cooperativa. Estos resultados están en clara consonancia con Thorp (2014), el cual establece que el género es determinante en la motivación hacia la práctica deportiva, y condiciona la misma en un estilo de vida saludable. El autor matiza que la asociación deportiva de los chicos está marcada por los aspectos competitivos, deportes individuales y deportes de equipo como fútbol y baloncesto, mientras que las chicas participan en actividades cooperativas y saludables como el aerobic, fitness o los paseos. Por lo tanto, no resulta extraño que el profesorado de educación física este muy influenciado por su práctica preferente en su itinerario vital y experiencia previa, mostrando una orientación más competi- 
tiva e individual en hombres, y más cooperativa en mujeres.

Son muchas y diferentes las preguntas que emergen a partir de este estudio. ¿De dónde surgen estas preferencias?, ¿Cómo se desarrollan?, ¿cuáles son realmente efectivas en el desempeño laboral, y en qué momento?, etc. El presente estudio ha desarrollado un instrumento que posee un gran potencial para conocer lo que los profesores prefieren en sus ambientes laborales, pero también puede servir como medio de conocer las preferencias de los futuros profesionales. En este sentido, como indicaban Price y Griggs (1985), en el plano formativo conocer estas preferencias posee diferentes aplicaciones, tales como aumentar la motivación de los futuros docentes al percibir que sus preferencias son consideradas en su formación. Favorecer el autoconocimiento así como la necesidad de conocer las preferencias participativas de sus propios alumnos (Ruiz et al., 2010), lo cual favorecería el desarrollo de estructuras de aprendizaje variadas que reconozcan las preferencias de los alumnos evitando conflictos de preferencias, y permitiría una verdadera consideración de las diferencias individuales.

Vivimos un momento en el que todo se centra en la promoción de los entornos cooperativos como la vía para favorecer el trabajo y el rendimiento, pero la realidad es más compleja y así debe ser contemplada. Las sesiones de educación física están llenas de situaciones en las que lo individual y lo colectivo se alternan y combinan, y donde lo cooperativo y lo competitivo se relacionan para alcanzar los objetivos planteados. Estas consideraciones son también aplicables al

\section{Referencias}

Anaya, D., y Suárez, J. M. (2010). Evaluación de la satisfacción laboral del profesorado y aportaciones a su mejora en orden a la calidad de la educación. Revista Española de Orientación y Psicopedagogia, 21(2), 293-294.

Balaguer, I., Castillo, I., y Tomás, I. (1996). Análisis de las propiedades psicométricas del Cuestionario de Orientación al Ego y a la Tarea en el deporte (TEOSQ) en su traducción al castellano. Psicológica, 17, 71-81.

Barrett, T. (2005). Effects of cooperative learning on the performance of sixth-grade physical education students. Journal of Teaching in Physical Education, 24, 88-102.

Byrne, B. M. (2008). Testing for multigroup equivalence of a measuring instrument: A walk through the process. Psicothema, 20(4), 872-882.

Cárdenas, M., Méndez, L. M., y González, M.T. (2013). Evaluación del desempeño docente, estrés y burnout en profesores universitarios. Revista Actualidades Investigativas en Educación, 14(1), 1-22.

Castro, M., Gómez, A., y Macazaga, A. (2014). Aprendizaje dialógico y grupos interactivos en educación física. Retos: Nuevas tendencias en Educación Física, Deporte y Recreación, 25, 174-179.

Caussidier, C., El Hage, F., Munoz, F., Remki, L., Larribi, R., Khzami, S. E., y Favre, D. (2011). In search of a health education model: teachers' conceptions in four Mediterranean countries. Global Health Promotion, 18(4), 5-15.

Cheung, G. W., y Rensvold, R. B. (2002). Evaluating goodness-of-fit indexes for testing measurement invariance. Structural Equation Modeling, 9(2), 233-255.

Coenders, G., Batista Foguet, J. M., y Saris, W. E. (2005). Temas avanzados en modelos de ecuaciones estructurales. Madrid: La Muralla.

Dyson, B. (2001). Cooperative learning in an elementary Physical Education program. Journal of Teaching in Physical Education, 20, 264-281.

Dyson, B. (2002). The implementation of cooperative learning in an elementary Physical Education program. Journal of Teaching in Physical Education, 22, 69-85. desempeño profesional en el que no todo es cooperativo o competitivo, existen otras dimensiones que es necesario explorar, y otras estrategias necesarias de ser desarrolladas. Para un responsable de un Seminario o Departamento puede ser de gran utilidad conocer mejor las influencias recíprocas que en el entorno laboral se dan entre los diferentes componentes de un Seminario o Departamento, para un profesional es imprescindible conocer qué piensan sus alumnos sobre las estructuras de aprendizaje que propone y contemplar las mutuas influencias que pueden darse en el seno de la clase (Prados, Cubero y de la Mata, 2010).

En conclusión, el presente estudio presenta un instrumento que favorece que los profesionales, o los futuros profesionales, puedan conocer sus preferencias de interacción, analizar las posibilidades que estas preferencias tienen e incluso optar por aprender otras preferencias que no son las suyas. Todo esto favorecerá una reflexión sobre sus procesos cognitivos y los resultados que obtiene de ellos, favoreciendo su desarrollo metacognitivo.

Como limitación del presente estudio, es su concreción en una profesión y en una titulación específica. Este hecho se convierte en un elemento a considerar ya que como Monroy y Hernández (2014) destacan la titulación que se posee es un factor que puede influir en las tareas, contenidos y estructuras de aprendizaje que los profesionales pueden proporcionar a sus alumnos, y probablemente en sus preferencias personales de interacción laboral.

Dyson, B., y Strachan, K. (2000). Cooperative learning in a high school physical education program. Waikato Journal of Education, 6, 19-37.

Dyson, B., y Grineski, S. (2001). Using cooperative learning structures in physical education. Journal of Physical Education, Recreation \& Dance, 72(2), 28-31.

Elosua, P. (2005). Evaluación progresiva de la invarianza factorial entre las versiones original y adaptada de una escala de autoconcepto. Psicothema, 17(2), 356-362.

Fernández, E., Sánchez, F., y Salinero, J. J. (2008). Validación y adaptación de la escala PACES de disfrute com la práctica de la actividad física para adolescentes españolas. Psicothema, 20(4), 890-895.

Flores, G., Prat i Grau, M., y Soler Prat, S. (2014). La voz del profesorado de educación física sobre su formación académica ante la realidad multicultural. Revista Electrónica Interuniversitaria de Formación del Profesorado, 17(2), 183-199

García, H. (1997). La formación del profesorado en Educación Física: problemas y expectativas. Barcelona: Inde.

González, G., y Barba, J. J. (2014). Formación permanente y desarrollo de la identidad reflexiva del profesorado desde las perspectivas grupal e individual. Profesorado. Revista de Currículum y Formación del Profesorado, 18(1), 397-412.

Johnson, D.W. (1981). Student-student interaction: The neglected variable in education. Educational Researcher, 10, 5-10.

Johnson, D.W., Johnson, R.T., y Holubec, E.J. (1994). Cooperative learning in the classroom. Virginia: Association for Supervision and Curriculum Development.

Johnson, D., Maruyama, G., Johnson, R., Nelson, D., y Skon, L. (1981). Effects of cooperative, competitive and individualistic goal structures on achievement: A meta-analysis. Psychological Bulletin, 89, 47-62. 
Monroy, F., y Hernández Pina, F. (2014). Factors affecting student approaches to learning. A systematic review. Educación XX1, 17(2), 105 124. doi: $10.5944 /$ educ $x \times 1.17 .2 .11481$

Morrison, G. M., Wakefield, P., Walker, D., y Solberg, S. (1994). Teacher preferences for collaborative relationships: Relationship to efficacy for teaching in prevention-related domains. Psychology in the Schools, 31(3), 221-231.

Nunnally, J. C. (1978). Psychometric theory ( $2^{\mathrm{a}}$ ed.). Nueva York: McGraw-Hill.

Orlick, T. (1978a). Winning through cooperation: competitive insanity, cooperative alternatives. Washington, DC: Accropolis Press.

Orlick, T. (1978b). The cooperative sports and games book: Challenge without competition. New York, NY: Pantheon Books.

Orlick, T. (1988). The second cooperative sports \& games book. Barcelona: Pantheon Books.

Orlick, T. (2006). Cooperative games and sports: Joyful activities for everyone. Champaign, IL: Human Kinetics.

Pashler, H., McDaniel, M., Rohrer, D., y Bjork, R. (2009). Learning styles. Concepts and evidences. Psychological Science in the Public Interest, 9(3), 105-119.

Peiró, C. (1999). La teoría de las perspectivas de meta y la educación física. Un estudio sobre los climas motivacionales. Revista de Psicología Social Aplicada, 9 (1), 25-44.

Polvi, S., y Telama, R. (2000). The use of cooperative learning as a social enhancer in physical education. Scandinavian Journal of Educational Research, 44(1), 105-115.
Prados, M., Cubero, M., y de la Mata, M. (2010). ¿Mediante qué estructuras interactivas se relacionan profesorado y alumnado en las aulas universitarias?. Electronic Journal of Research in Educational Psychology, 8(1), 163-194.

Pryce, G. E., y Briggs, S. A. (1985). Counselling college students through their individual learning styles. Ann Arbor, Michigan: University of Michigan, ERIC. Texto libre: http://fi les.eric.ed.gov/ fulltext/ED265455.pdf

Ruiz, L. M., Graupera, J. L., Moreno, J. A., y Rico, I. (2010). Social preferences for learning among adolescents in Secondary Physical Education. Journal of Teaching in Physical Education, 29, 3-20.

Sánchez-Oliva, D., Sánchez-Miguel, P. A., Miguel-Leo, F. M., Amado, D., y García-Calvo, T. (2013). Desarrollo y validación de un cuestionario para analizar la percepción de comportamientos positivos en las clases de educación física. Cultura y Educación, 25(4), 1-9.

Tani, G. (2013). Professional preparation in physical education: changing labour market and competence. Motriz: Revista Educaçao Física, 19(3), 552-557.

Thorp, J. L. (2014). Enganging students in physical education: recommendations for secondary programs. Strategies: A Journal for Physical and Sport Educators, 26(1), 8-13.

Worthington, R. L., y Whittaker, T. A. (2006). Scale development research: A content analysis and recommendations for best practices. The Counseling Psychologist, 34(6), 806-838.

Yaylaci, F. (2011). Evaluation of professional perceptions by physical education teachers and pre-service teachers. World Applied Sciences Journal, 12(10), 1760-1772.

(Articulo recibido: 13-11-2014; revisado: 09-02-2015; aceptado: 10-03-2015) 